\title{
Development of Nowcast of Atmospheric Ionizing Radiation for Aviation Safety (NAIRAS) model
}

\author{
Christopher J. Mertens ${ }^{1}$, W. Kent Tobiska ${ }^{2}$, David Bouwer ${ }^{2}$, Brian T. Kress ${ }^{3}$, Michael J. \\ Wiltberger ${ }^{4}$, Stanley C. Solomon ${ }^{4}$, and John J. Murray ${ }^{1}$ \\ ${ }^{1}$ NASA Langley Research Center \\ Hampton, Virginia 23681 \\ ${ }^{2}$ Space Environment Technologies, Inc. \\ Pacific Palisades, California \\ ${ }^{3}$ Dartmouth College \\ Hanover, New Hampshire 03755 \\ ${ }^{4}$ High Altitude Observatory, National Center for Atmospheric Research \\ Boulder, Colorado 80301
}

\begin{abstract}
In this paper an overview is given of the development of a new nowcast prediction of aircrew radiation exposure from both background galactic cosmic rays (GCR) and solar energetic particle events (SEP) that may accompany solar storms. The new air-crew radiation exposure model is called the Nowcast of Atmospheric Ionizing Radiation for Aviation Safety (NAIRAS) model. NAIRAS will provide global, data-driven, real-time radiation exposure predictions of biologically harmful radiation at commercial airline altitudes. Observations are utilized from the ground (neutron monitors), from the atmosphere (the NCEP Reanalysis and NCEP Global Forecasting System), and from space (NASA/ACE and NOAA/GOES). Atmospheric observations provide the overhead shielding information and the ground- and space-based observations provide boundary conditions on the incident GCR and SEP particle flux distributions for transport and dosimetry simulations. Exposure rates are calculated using the physics-based HZETRN (High Charge and Energy Transport) code. Recent progress in the model implementation is reported and examples of the model results are shown for a representative high-energy SEP event during the Halloween 2003 superstorm, with emphasis on the high-latitude and polar region. The suppression of the geomagnetic cutoff rigidity during these storm periods and their subsequent influence on atmospheric radiation exposure is characterized.
\end{abstract}

\subsection{System Architecture}

In its first year of performance, using rapid-prototyping methods, Space Environment Technologies (SET) has used team member (stake-holder) participation to identify the critical input data streams. As a result, the NAIRAS high-level design architecture 
(Figure 1) is now a working prototype on an operational server. The primary and secondary (redundant) input data sources (Table 1 and Figure 2) are now being gathered continuously in a low cadence demonstration mode and provided from the current epoch back through the past 4 days.

The risks for operationally incorporating redundant data streams have been identified with the most significant outlier being the lack of availability of solar wind data in case the ACE satellite goes off line. Both SET and the NOAA Space Weather Prediction Center (SWPC) looked at this issue and the team concluded that the ENLIL plus cone model may be a possible alternative, with the penalty of higher uncertainties, to supply solar wind parameters in the event of ACE data loss. Other possible approaches are to implement a data gap-fill algorithm, or calculate geomagnetic cutoff rigidities using a simpler magnetospheric magnetic field model that utilizes a readily available geomagnetic activity index (such as kp or Dst, for example). Each of these approaches will be examined and evaluated in the coming months.

The SET preliminary Requirements Specification Document has been issued as SET_report_1.pdf and it complements the I/O requirements along with data definitions that have been established by the PI and SET team members as outlined in Figure 2. Input data formats have been specified in algorithms and the data are being stored for 4 days in the database. A significant effort was required by SET to learn the format of NCAR/NCEP and NCEP/NWS grib data files for tropospheric and stratospheric parameters. The team was successful in interpreting and operationally incorporating these data. The current epoch temperature at a given pressure level (examples of surface, troposphere, and stratosphere) can be viewed at the http://spacewx.com site, SpWx Now:Atmosphere Now menu items. The GOES particle data posed unique challenges: SET was the beta-tester for the SPWC E-SWDS system (http://spacewx.com, Innovation:E-SWDS menu item) whereby GOES data are now extracted directly from NOAA servers and not the web portal. In the event E-SWDS fails, as has happened in one or two cases as the system started up, SET's servers automatically go to the NOAA web portal to retrieve data at a penalty of longer latency.

In addition to the operational server prototype, SET has established a password-protected team website (http://sol.spacenvironment.net/ raps_ops/index.php) and a public website (http://sol.spacenvironment.net/ nairas/index.html) for NAIRAS. Both sites will be used for evolving NAIRAS to the next stage of test case model runs that access the SET database for input data, and then provide output files to be picked up by the SET server for deposit to the database. A task in the next year will be for each team member to help define the requirements/data formats for his/her respective area of contribution and the test cases will help us do this. 


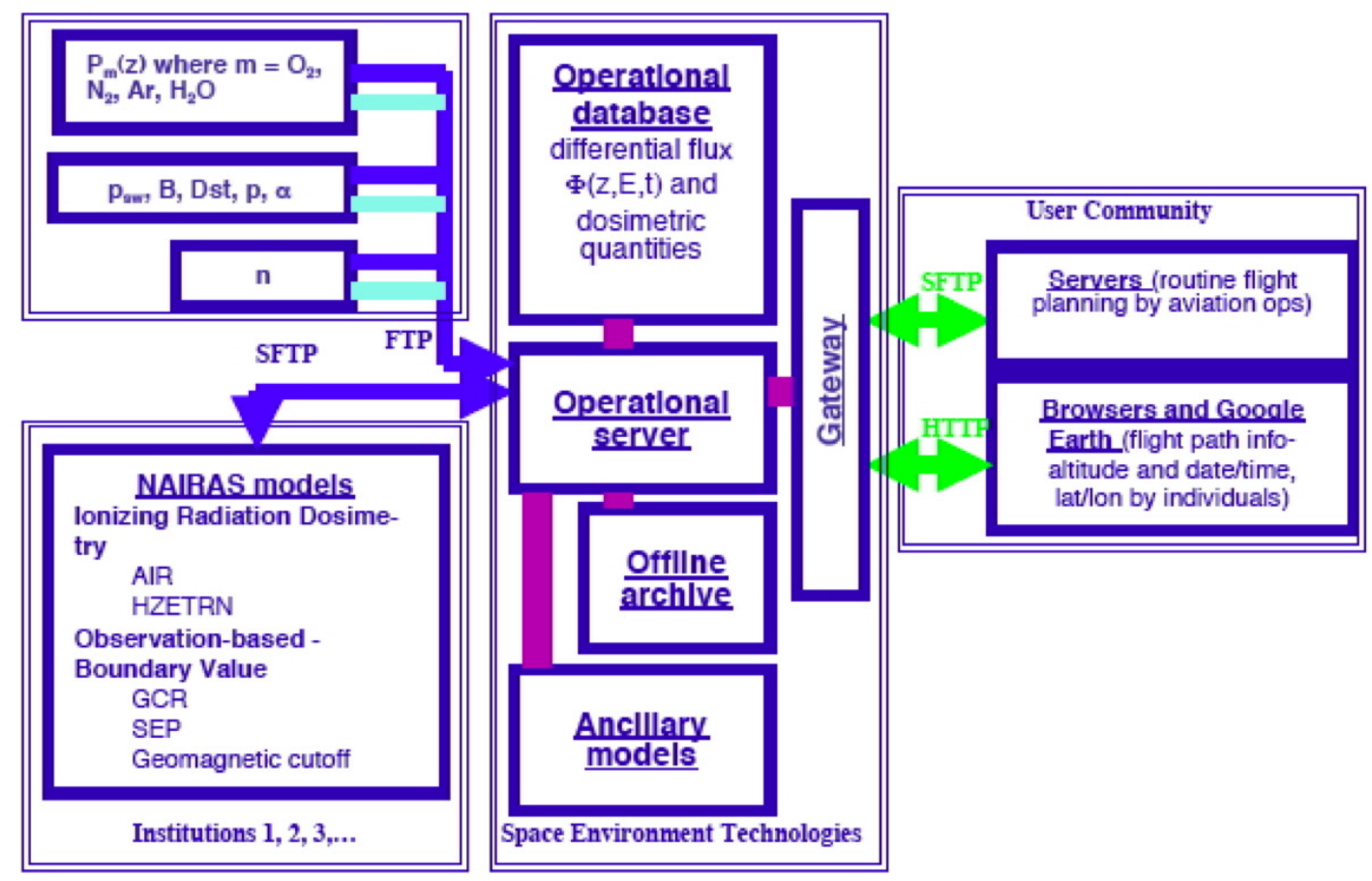

Figure 1: NAIRAS operational distributed network system architecture.

\subsection{Model Development}

Since evasive maneuvers and/or critical flight path planning of commercial and business aircraft may be warranted during large solar energetic particles (SEP) events, our initial effort has focused on the nowcast of SEP atmospheric radiation exposure. NAIRAS model predictions of atmospheric SEP radiation exposure are driven by measurements from the atmosphere and from space. Real-time satellite ion flux measurements are used to derive the SEP proton and alpha fluence spectra incident at the top of the atmosphere. The geomagnetic field (internal field plus magnetospheric contributions) filters the incident SEP fluence spectra by deflecting the lower energy particles back out to space. This spectral filtering effect is quantified by a canonical variable called the geomagnetic cutoff rigidity. Satellite measurements of solar wind dynamical pressure and the interplanetary magnetic field (IMF) are used to characterize the magnetospheric contributions to the cutoff rigidity. Global meteorological measurements combined with data assimilation and forecasts models are used to predict atmospheric depth as a function of altitude. In the subsections below, we describe the data input and space environment models developed to derive: (1) the incident SEP fluence spectra, (2) the geomagnetic cutoff rigidity, and (3) atmospheric depth as a function of altitude. Finally, we briefly describe significant algorithm improvements to HZETRN, the physics-based deterministic transport code used to propagate the incident SEP particles through the atmosphere and calculate the radiation exposure. 


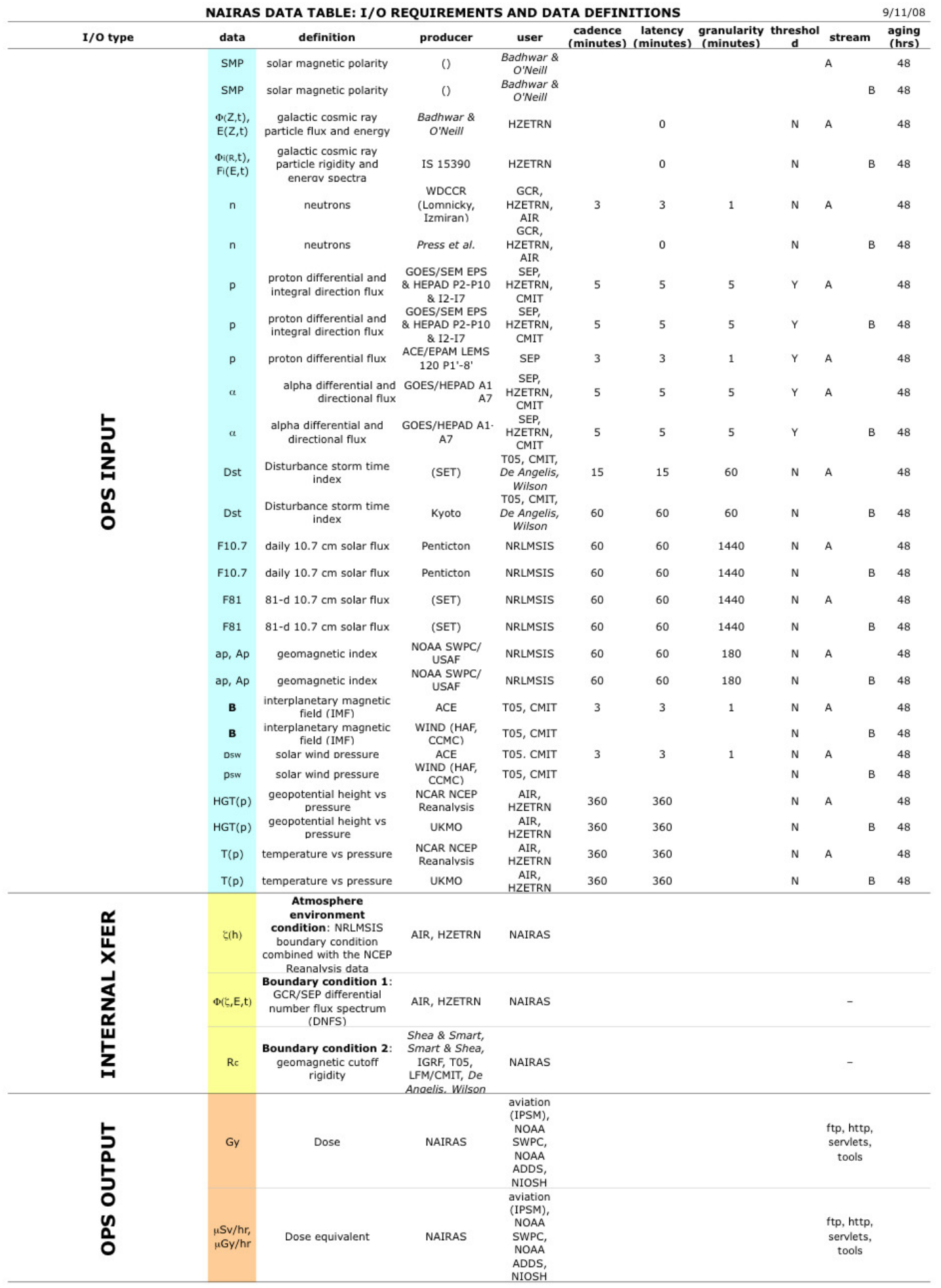

Figure 2: NAIRAS primary and secondary (redundant) input data sources and definitions, output data definitions, and data/model coupling. 


\subsection{SEP Spectral Fluence}

The NAIRAS model initially assumes a double power-law form for the SEP fluence spectrum and derives fit parameters by a non-linear least-square fit to differentialdirectional fluence measurements [Mewaldt et al., 2005]. The spectral fitting algorithm uses a Marquardt-Levenberg iteration technique [Brandt, 1999]. If the double power-law spectrum fails to converge to the measurement data, the fitting procedure is restarted and the so-called Ellison-Ramaty spectral form is assumed [Ellison and Ramaty, 1985]. The algorithm has been developed and tested on a number of storm periods.

The NAIRAS model utilizes available real-time measurements of proton and alpha differential-directional particle flux $\left(\mathrm{cm}^{2} \text {-sr-sec-MeV/n }\right)^{-1}$ for the SEP spectral fitting described above. Fluence is obtained by time-integrating the particle flux measurements. Low-energy proton data are obtained from the Electron, Proton, and Alpha Monitor (EPAM) instrument onboard the NASA Advanced Composition Explorer (ACE) satellite [Gold et al., 1998]. EPAM is composed of five telescopes and we use the LEMS120 (Low-Energy Magnetic Spectrometer) detector, which measures ions at 120 degrees from the spacecraft axis. LEMS120 is the EPAM low-energy ion data available in real-time, for reasons described by Haggerty et al. [2006]. The other proton channels used in the SEP spectral fitting algorithm are obtained from NOAA's Geostationary Operational Environmental Satellite (GOES) Space Environment Monitor (SEM) measurements. The Energetic Particle Sensor (EPS) and the High Energy Proton and Alpha Detector (HEPAD) sensors on GOES/SEM measure differential-directional proton flux [Onsager et al., 1996]. We also generate additional differential proton measurement channels by taking differences between the EPS integral proton flux channels. The channels used to derive SEP alpha fluence spectra are also obtained from EPS measurements. We use 5minute averaged ACE and GOES data to derive the SEP fluence spectra.

Figure 3 shows the time variation of the flux measurements listed in Table 2 used to derive SEP fluence spectra for the Halloween 2003 storm period. The top panel displays the ACE low-energy proton flux measurements. The next two panels show the GOES-11 EPS and HEAD proton flux spectra, and the integral proton flux measurements, respectively. By definition, a SEP event occurs when the $>10 \mathrm{MeV}$ integral proton flux exceeds 10 proton flux units (pfu: $\mathrm{cm}^{-2} \mathrm{sr}^{-1} \mathrm{sec}^{-1}$ ). The SEP event threshold is denoted by the horizontal line on the integral proton flux panel. There are a total of five SEP events during the Halloween 2003 storm period, which are denoted by the vertical lines in all panels in Figure 3. Different line styles are used to bound each of the five events, and the event number is shown between the vertical lines. Note that the onset of event 3 doesn't follow the conventional SEP threshold definition. It is clear from the integral proton flux that two events overlap: event 3 arrives before event 2 decreases below the SEP threshold level. However, there is an important distinguishing feature between the two events. That is, the beginning of our definition of event 3 is accompanied by a sudden increase in high-energy protons associated with the arriving SEP event, as noted by the sudden 


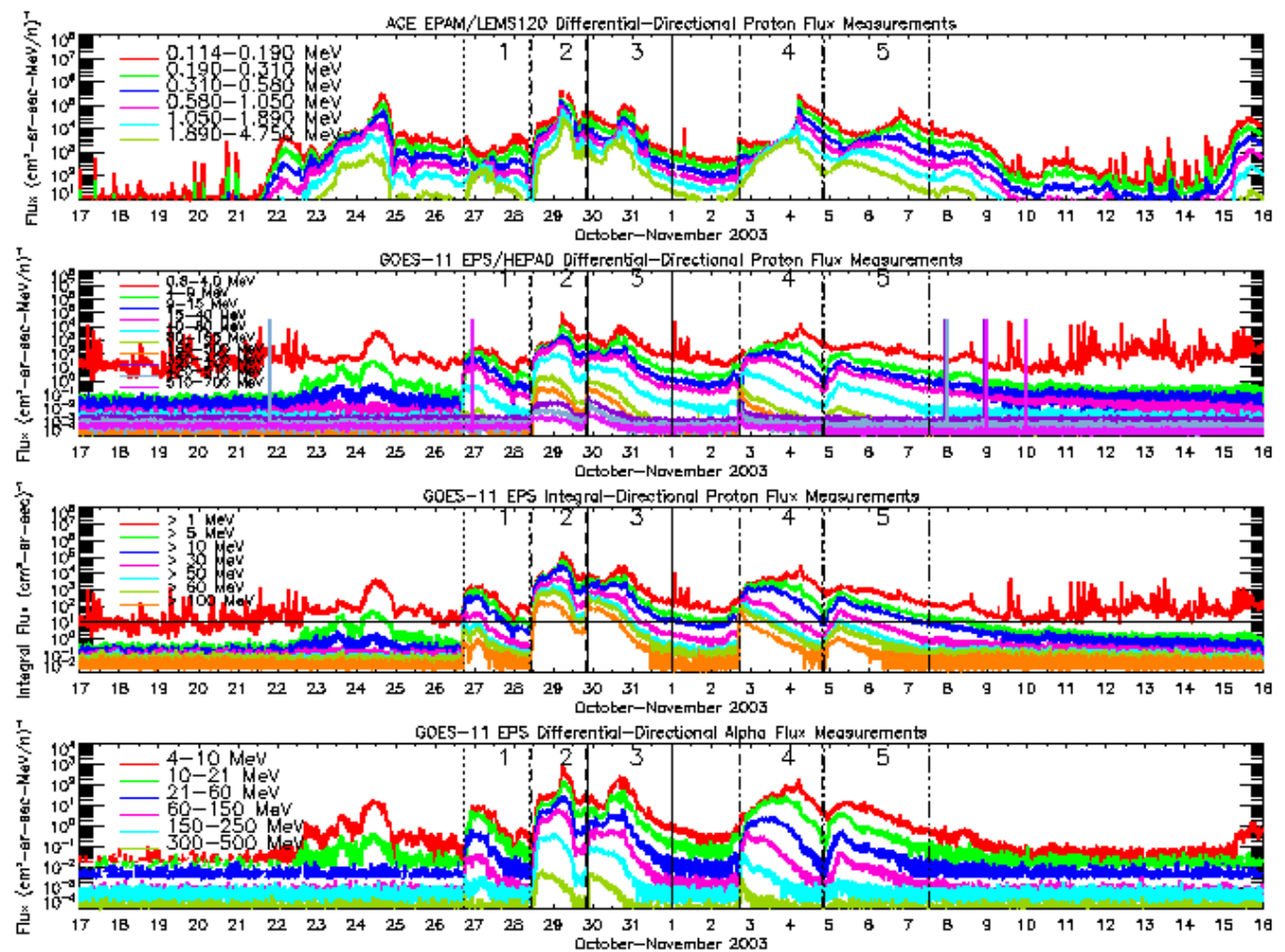

Figure 3: Proton and alpha flux measurements used to derive the SEP fluence spectra. Row 1: ACE EPAM/LEMS120 differential-directional proton flux measurements. Row 2: GOES-11 EPS and HEPAD differential-directional proton flux measurements. Row 3: GOES-11 EPS integraldirectional proton flux measurements. Row 4: GOES-11 EPS differential-directional alpha flux measurements. The different styled vertical lines bound the five SEP events during the Halloween 2003 solar-geomagnetic storm period, which are numbered in all panels. The horizontal line in Row 3 indicates the SEP threshold for the $>10 \mathrm{MeV}$ integral proton flux channel.

increase in the 510-700 MeV differential proton flux measurements in Figure 3. Partitioning the simultaneous SEP events 2 and 3 into separate events is useful for our initial studies, since the high-energy portion of the differential proton flux distribution penetrates deeper in the atmosphere.

We analyzed atmospheric radiation exposure during SEP event 3 in our initial investigations, because of the comparatively large high-energy component of the proton spectrum associated with this event. In order to isolate geomagnetic effects, we derived the spectra shown in Figure 4.The horizontal lines in Figure 4 are the event-averaged differential-directional fluence measurements. The widths of the horizontal lines correspond to the energy width of the measurement channels. The black lines are the proton and alpha fluence spectra derived using the double power-law spectrum and fitting technique describe above. 


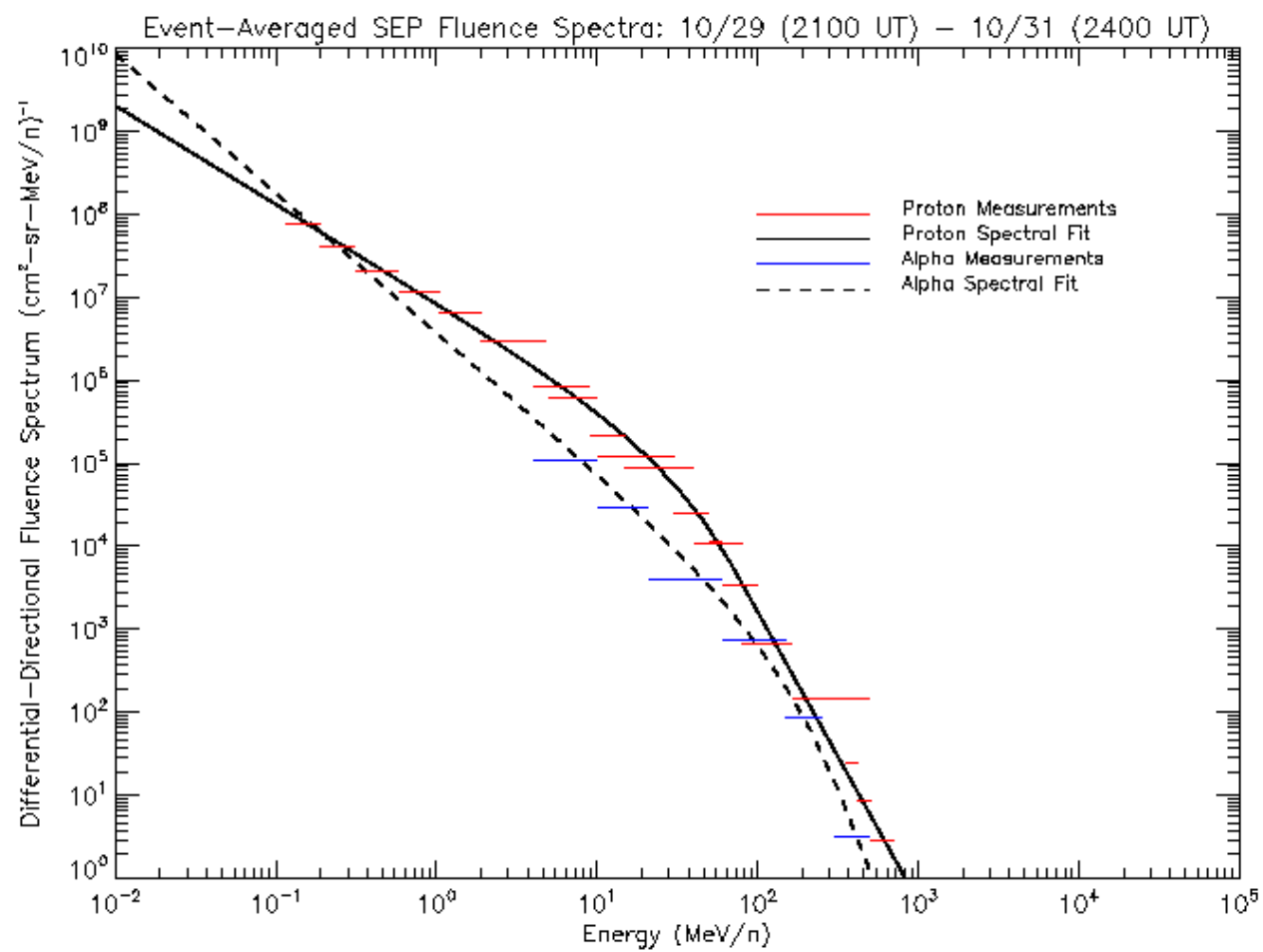

Figure 4: Event-averaged SEP fluence spectra for event 3 [10/29/2003 (2100 UT) - 10/31/2003 (2400 UT)].

\subsection{Geomagnetic Cutoff Rigidity}

The NAIRAS cutoff rigidities are calculated from code developed by the Center for Integrated Space Weather Modeling (CISM) at Dartmouth College. The CISMDartmouth geomagnetic cutoff model can be run using several different empirical and physics-based models [Kress et al., 2004]. In particular, the specification of the geomagnetic field due to Earth's internal field source is provided by the International Geomagnetic Reference Field (IGRF) model [Langlais and Mandea, 2000]. The magnetospheric current systems are also important contributors to Earth's total geomagnetic field. The real-time dynamical response of the magnetospheric magnetic field to solar wind conditions and interplanetary magnetic field (IMF) can be provided by the semi-empirical T05 model [Tsyganeko and Sitnov, 2005], or by the Lyon-FederMobarry (LFM) global MHD simulation code [Lyon et al., 2004]. Routines were developed and tested to couple the geomagnetic cutoff model with the different magnetic field models. 

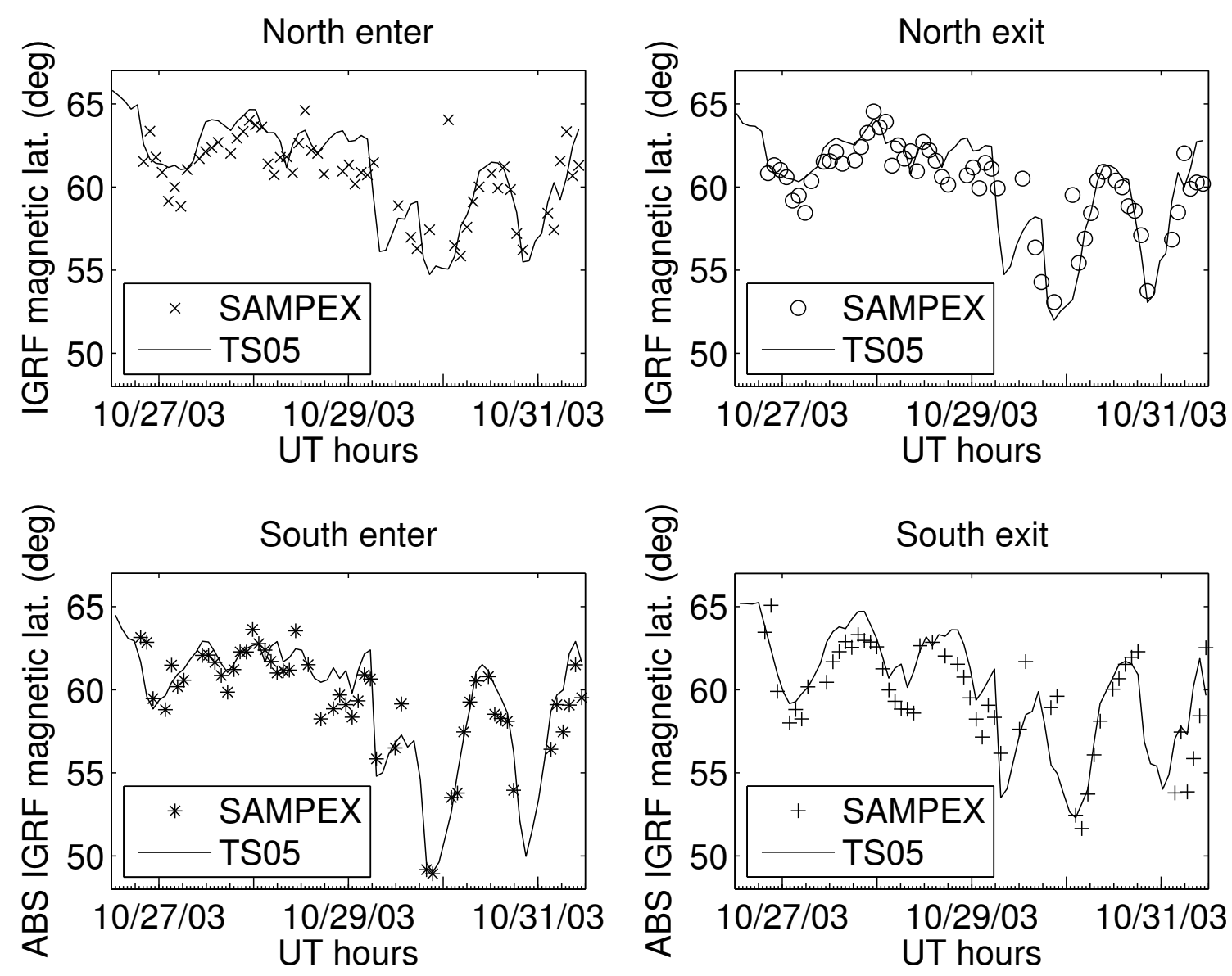

Figure 5: The four panels show comparisons between cutoff latitudes determined in the T05 geomagnetic field model and cutoff latitudes extracted from SAMPEX/PET energetic particle data during 28-31 October (Halloween) 2003 storms. Cutoff latitudes as SAMPEX enters and exists the north and south polar cap regions are shown separately.

The LFM MHD code may be run as a stand alone model or coupled with other geospace models currently under development within CISM. For example, the LFM magnetospheric magnetic fields may be coupled with the Thermosphere-Ionosphere Nested Grid (TING) model [Wang et al., 1999]and/or with the Rice Convection Model (RCM) [Toffoletto et al., 2004], which models the ring current. The semi-empirical T05 model provides more accurate cutoff rigidities than the stand alone LFM MHD model, as determined by comparisons with satellite observations during a Halloween 2003 geomagnetic storm. Figure 5 shows comparisons between cutoff latitudes for $\sim 20 \mathrm{MeV}$ protons computed using the T05 model and measured by the proton electron telescope (PET) instrument on the Solar Anomalous and Magnetospheric Particle Explorer (SAMPEX) satellite [Baker et al., 1993].

The agreement between SAMPEX/PET measurements and the T05 model in Figure 5 are quite good. The reason the physics-based LFM MHD model doesn't calculate cutoffs as accurate as the semi-physical T05 model is mainly due to the lack of a full kinetic description of the ring current in the MHD model, which typically causes the LFM fields to be too high. This is evident in Figure 6, which compares geomagnetic cutoff rigidities 
calculated along three representative high-latitude commercial flight routes from the IGRF, T05, and LFM MHD magnetic field models. The small differences in cutoffs using the LFM MHD model between quiet and geomagnetic storm conditions is indicative of an inadequate modeling of the ring current build-up during the geomagnetic storm.
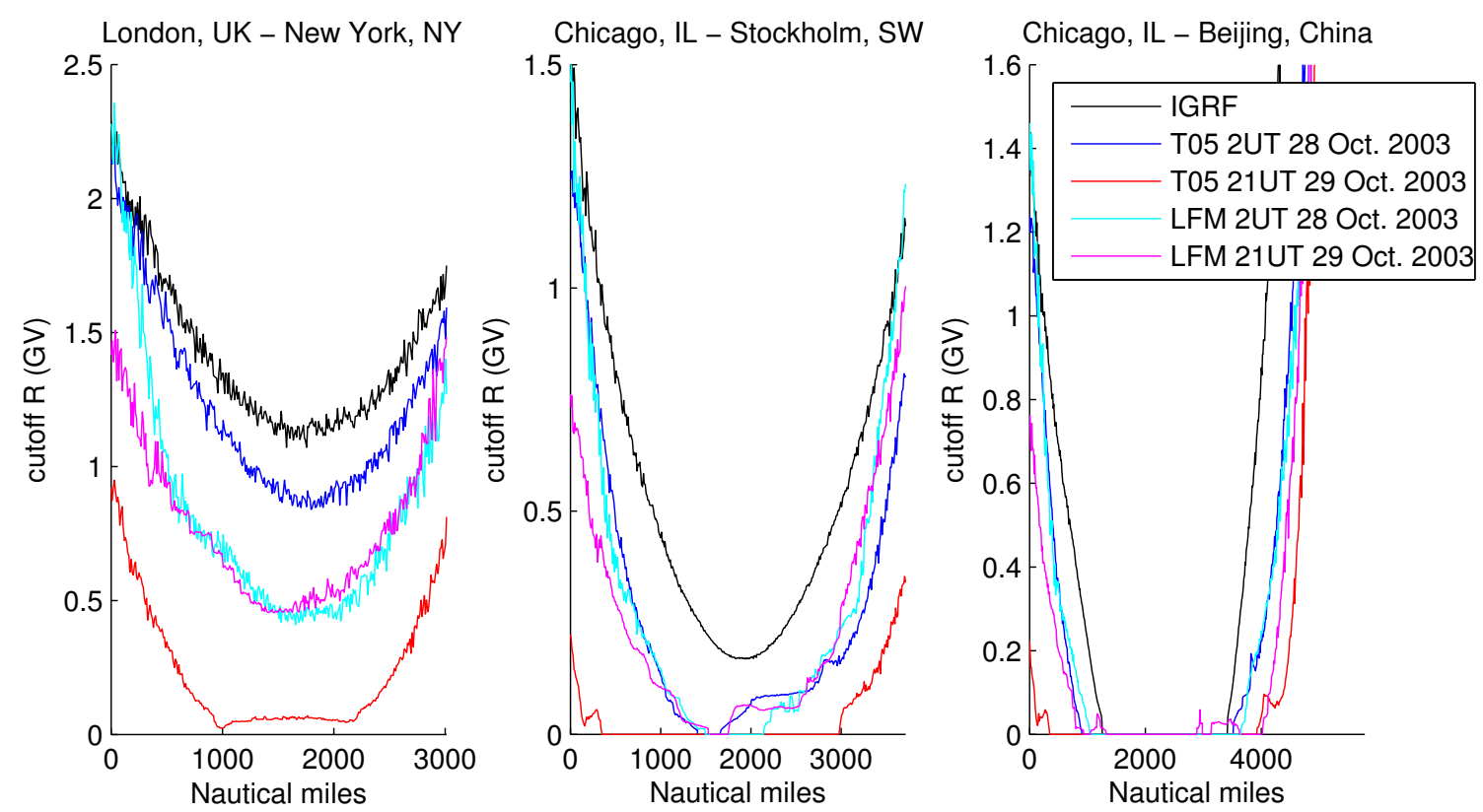

Figure 6: Geomagnetic cutoff rigidities along three high-latitude commercial flight routes computed in three different magnetic field models: IGRF, T05, and LFM MHD. The October 28 (0200 UT) calculations represent a magnetically quiet time, while the October 29 (2100 UT) calculations occur during a geomagnetic storm.

We anticipate that the fully coupled LFM-RCM-TING model currently under development will significantly improve the simulations of cutoff rigidities compared to the stand along LFM MHD model. Furthermore, the physics-based LFM-RCM-TING model will be able to incorporate short time-scale dynamics not included in empirical magnetospheric magnetic field models.

When the code development within CISM reaches sufficient maturity, we will assess the influence of short time-scale magnetospheric dynamics on the atmospheric ionizing radiation field using the fully coupled LFM-RCM-TING model. For the present work, the simulated real-time geomagnetic cutoff rigidities are calculated using the T05 model, and using the IGRF model for comparison.

Figure 7 shows the vertical cutoff rigidity over the northern hemisphere for three different models of the geomagnetic field during the Halloween 2003 storm period. The left column is cutoff rigidity computed using the IGRF field. Since total flight-path exposure at aviation altitudes do not change significantly $(<\sim 1 \%)$ for cutoffs less than $0.05 \mathrm{GV}$, we set the cutoffs to zero at geographic locations poleward of the $0.05 \mathrm{GV}$ contour (see the bold-white $0.05 \mathrm{GV}$ color contour in Figure 7). The middle column in Figure 7 shows the cutoff rigidities computed using the T05 field under geomagnetically quiet conditions, October 28 (0200 UT), prior to the onset of the Halloween 2003 SEP 
event 3 . One can see that even during magnetically quiet conditions, the cutoff rigidities predicted from the T05 field are lower than predicted from the IGRF field, and the polar cap region (i.e., inside the bold-white $0.05 \mathrm{GV}$ contour in Figure 7) is expanded to lower latitudes. A weaker field predicted by the T05 model, compared to IGRF, is due in part to the diamagnetic effect of the magnetospheric ring current included in the T05 model. Lower cutoff rigidities correspond to less momentum shielding and higher radiation exposure levels. The right column in Figure 7 shows the cutoff rigidities during peak geomagnetic storm conditions, October 29 (2100 UT), during SEP event 3. The cutoffs are lower at all latitudes compared to the two previous simulations, and the polar cap region has expanded to much lower latitudes than during the magnetically quiet period.

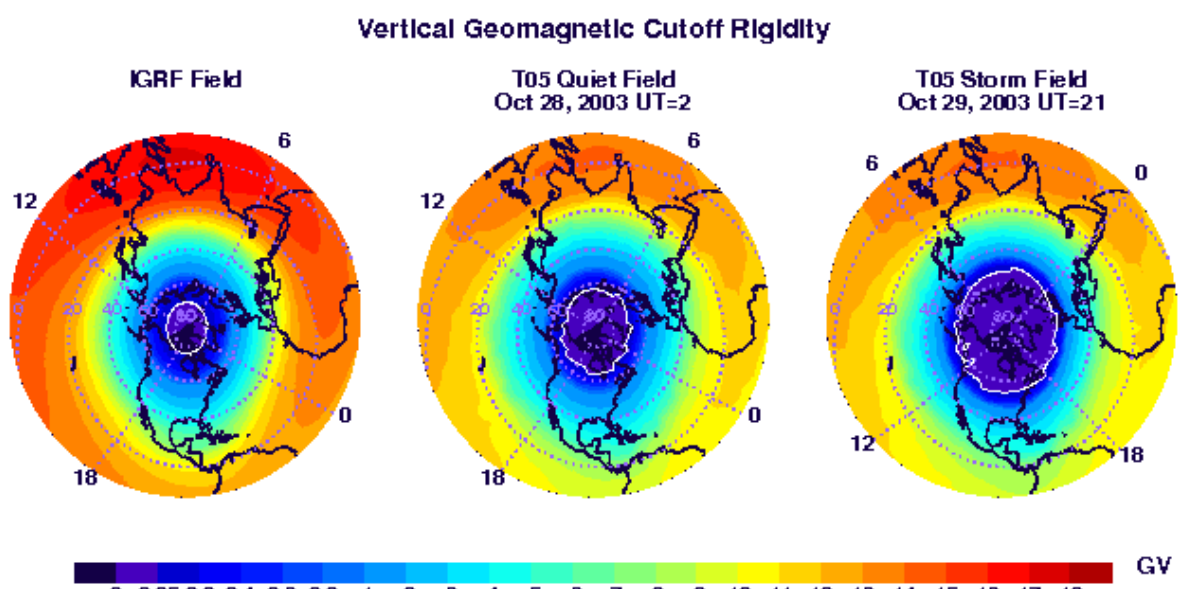

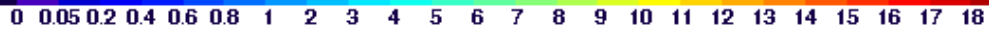

Figure 7: Simulated vertical geomagnetic cutoff rigidity shown over the northern hemisphere in October 2003. Also shown are the magnetic latitude circles and the meridians at $0,6,12,18$ magnetic local time.

The difference in cutoffs between storm and quiet conditions is shown in Figure 8 . The cutoffs were calculated using the T05 model. The magnetically quiet period is the same as above, October 28 (0200 UT), 2003. The top panel shows the cutoff difference between the magnetically quiet time and the arrival of an interplanetary shock at the magnetosphere on October 29 (0612 UT), 2003. The bottom panel shown the storm-quiet cutoff difference when the Disturbed Storm Time (Dst) index is near its minimum on October 29 (2100 UT), 2003. The cutoffs are most suppressed at mid-latitudes during the 
night. The storm-quiet cutoff difference can be as much as $\sim 1 \mathrm{GV}$, which has a significant effect on radiation exposure.

\section{Difference between shock arrival and quiet cutoffs}

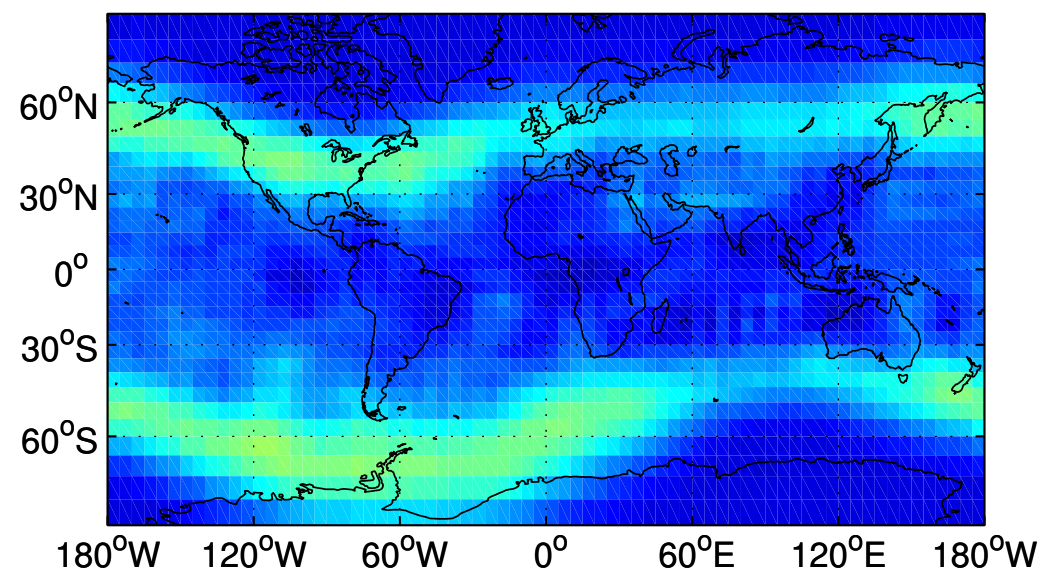

Difference between minimum Dst and quiet cutoffs

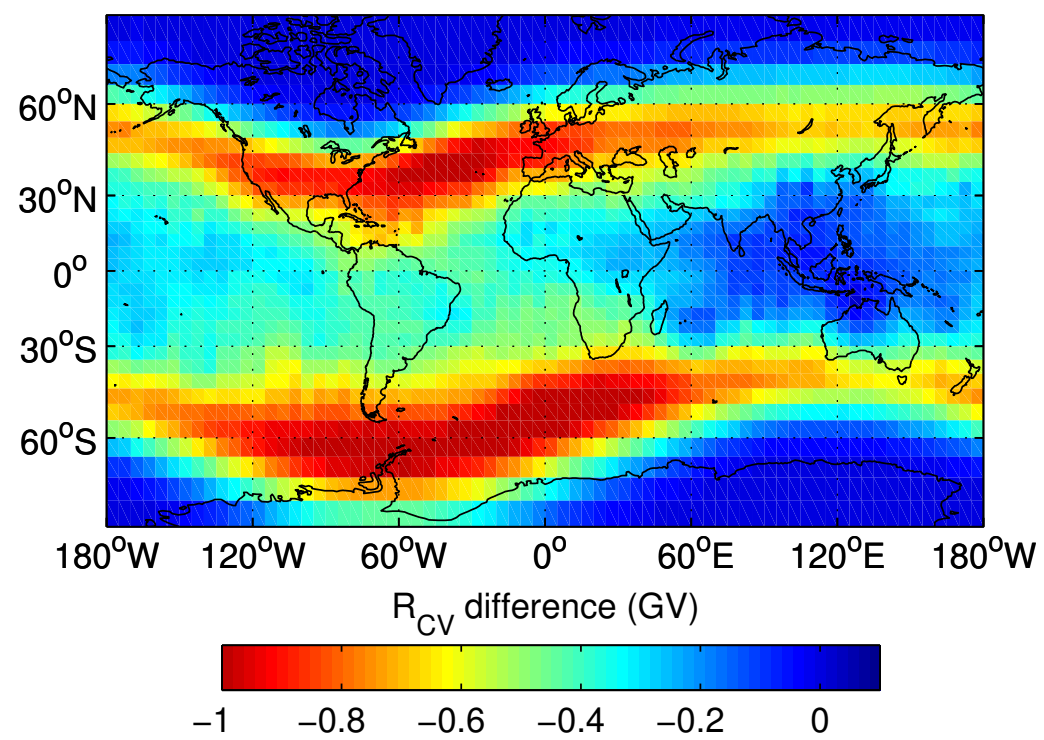

Figure 8: Storm-quiet geomagnetic cutoff rigidities differences calculated using the T05 magnetospheric magnetic field model. The magnetically quiet time cutoffs were calculated on October $28(0200$ UT), 2003. The storm cutoffs in the top panel were computed on October $29(0612)$, 2003. The storm cutoffs in the bottom panel were computed on October 29 (2100 UT), 2003.

An important aspect of these model studies is our assessment of the impact of the changes in cutoff rigidity due to the magnetospheric field effect on atmospheric radiation exposure, and the identification of the need for accurate and computationally efficient geomagnetic cutoff rigidity models with solar wind-magnetospheric dynamical responses 
included. The $\sim 1 \mathrm{GV}$ suppression in cutoff at mid-latitudes during a geomagnetic storm means that high-level SEP radiation exposure normally confined to the polar cap region will be extended to mid-latitudes. More details of these findings are summarized in section 3.0.

Considerable effort was applied to quantifying the minimum cutoff required in the numerical simulations. Small cutoff rigidities require more computational time since the time step in the numerical charged particle trajectory calculations in the geomagnetic field are a fraction of the gyroperiod. Initially, we found that differences in accumulated exposure for typical high-latitude flight paths are within $1 \%$ if the simulated cutoff rigidity is set to zero for rigidities less than $0.05 \mathrm{GV}$. However, we found discontinuous features in the exposure rates along the flight paths by setting the minimum cutoff to 0.05 $\mathrm{GV}$. These discontinuities are suppressed if the minimum cutoff rigidity is set to 0.01 GV.

\subsection{Meteorological Data}

The atmospheric itself provides shielding from incident charged particles. The shielding of the atmosphere at a given altitude depends on the overhead mass. Sub-daily global atmospheric depth is determined from pressure versus geopotential height and pressure versus temperature data derived from the National Center for Environmental Prediction (NCEP) / National Center for Atmospheric Research (NCAR) Reanalysis 1 project [Kalnay et al., 1996]. The NCAR/NCEP Reanalysis 1 project uses a state-of-the-art analysis/forecast system to perform data assimilation using past data from 1948 to the present. The data products are available $4 \mathrm{x}$ daily at $0,6,12$, and $18 \mathrm{UT}$. The spatial coverage is 17 pressure levels in the vertical from approximately the surface $(1000 \mathrm{hPa})$ to the middle stratosphere ( $10 \mathrm{hPa}$ ), while the horizontal grid is $2.5 \times 2.5$ degrees covering the entire globe.

NCEP/NCAR pressure versus geopotential height data is extended in altitude above 10 hPa using the Naval Research Laboratory Mass Spectrometer and Incoherent Scatter (NRLMSIS) model atmosphere [Picone et al., 2002]. NCEP/NCAR and NRLMSIS temperatures are smoothly merged at $10 \mathrm{hPa}$ at each horizontal grid point. NRLMSIS temperatures are produced at $2 \mathrm{~km}$ vertical spacing from the altitude of the NCEP/NCAR $10 \mathrm{hPa}$ pressure surface to approximately $100 \mathrm{~km}$. The pressure at these extended altitudes can be determined from the barometric law using the NRLMSIS temperature profile and the known NCEP/NCAR $10 \mathrm{hPa}$ pressure level, which assumes that the atmosphere is in hydrostatic equilibrium and obeys the ideal gas law. Finally, the altitudes and temperatures are linearly interpolated in log pressure to a fixed pressure grid from $1000 \mathrm{hPa}$ to $0.001 \mathrm{hPa}$, with six pressure levels per decade. The result from this step is pressure versus altitude at each horizontal grid point from the surface to approximately $100 \mathrm{~km}$.

Atmospheric depth $\left(\mathrm{g} / \mathrm{cm}^{2}\right)$ at each altitude level and horizontal grid point is computed by vertically integrating the mass density from a given altitude to the top of the atmosphere. The mass density is determined by the ideal gas law using the pressure and temperature at 
each altitude level. The result from this step produces a 3-D gridded field of atmospheric depth. Atmospheric depth at any specified aircraft altitude is determined by linear interpolation along the vertical grid axis in log atmospheric depth. Figure 9 shows the atmospheric pressure over the northern hemisphere at $11 \mathrm{~km}$ on October 29, 2003 (2100 UT). This is the atmospheric data used in the exposure rate calculations in section 3.0.

NCAR/NCEP Reanalysis 1: Pressure at $11 \mathrm{~km}$
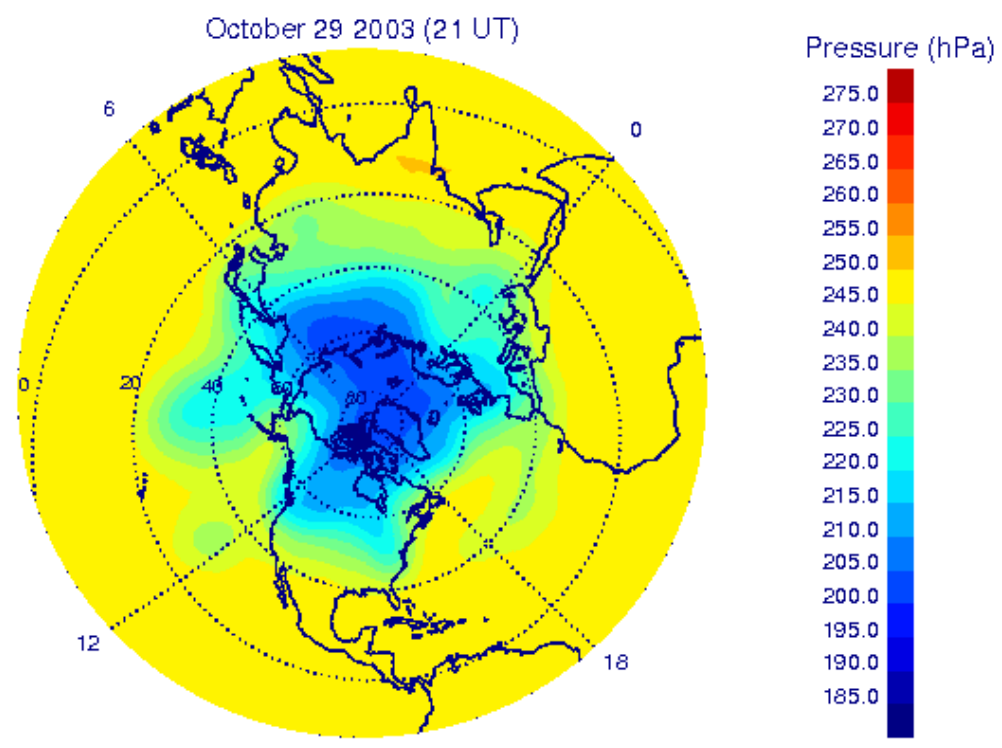

Figure 9: NCAR/NCEP Reanalysis 1 pressure levels at $11 \mathrm{~km}$ corresponding to the date/time of the largest geomagnetically disturbed period of SEP event $3(10 / 29 / 2003,2100$ UT). Also shown are the magnetic latitude circles and the meridians at $0,6,12$, and 18 magnetic local time.

In the process of developing the real-time data acquisition, we discovered an approximate two day delay in the availability of the NCAR/NCEP Reanalysis data. Through direct correspondence with the NCAR/NCEP Reanalysis team, it was apparent that the data latency could not be rectified. As a result, we have replaced our source of meteorological data from NCAR/NCEP Reanalysis to the NCEP Global Forecast System (GFS). The NCEP/GFS real-time data is updated every six hours, and at each update, a forecast is predicted every three hours, which extends out to the next 384 hours. Similar to the NCAR/NCEP Reanalysis data, NCEP/GFS provides temperature and geopotential height on fixed pressure surfaces. Thus, the methodology of extending the pressure versus geopotential height using the NRLMSIS model and calculating the real-time atmospheric depth as a function of altitude from the surface to $100 \mathrm{~km}$ is exactly the same as for the NCAR/NCEP Reanalysis data. However, new I/O software was developed to extract the meteorological data from the NCEP/GFS binary files. 


\subsection{Low-Energy Neutron Transport}

A recent update to HZETRN includes a directionally-coupled forward-backward lowenergy neutron transport algorithm, with coupling to light-ion transport [Slaba et al., 2008]. The new deterministic transport algorithm was compared to Monte Carlo codes HETC-HEDS, FLUKA, and MCNPX for the February, 1956 SEP event using the socalled Webber spectrum. The new HZETRN neutron transport calculations agreed very well with HETC-HEDS and reasonably well with FLUKA and MCNPX. The comparisons showed significant improvements over the previous forward-backward neutron transport model in HZETRN. The comparisons of the new deterministic neutron transport model with the Monte Carlo codes verified that coupling between low-energy neutrons and low-energy light-ion transport is not only necessary for accurate estimates of the fluence spectra, but for integrated quantities such as dose and dose equivalent as well.

The directionally-coupled forward-backward low-energy neutron transport algorithm recently incorporated in HZETRN represents a significant improvement for physicsbased deterministic atmospheric ionizing radiation transport. For atmospheric radiation exposure estimates, the new algorithm improved the accuracy of the contribution of backscattered neutrons. The largest contribution from backscattered neutrons to dose equivalent occurs in the region of typical commercial airline cruising altitudes.

\subsection{Analysis of High-Energy SEP Event}

Our initial model studies concentrated on predictions of SEP ambient dose equivalent rates and accumulated ambient dose equivalent along representative high-latitude commercial routes during the Halloween 2003 SEP event 3 [10/29 (2100 UT) - 10/31 (2400 UT)]. The incident SEP fluence and meteorological data were fixed in time in our calculations, which are given by the event-averaged fluence and atmospheric depthaltitude data shown in Figure 4 and Figure 9, respectively. On the other hand, we allowed the cutoff rigidity to vary in time along the flight trajectories, according to the magnetospheric magnetic field response to the real-time solar wind and IMF conditions. A primary objective of this study was to diagnose geomagnetic storm effects on SEP atmospheric radiation exposure. In the near future, we will allow the SEP fluence, cutoff rigidity, and atmospheric depth-altitude to all vary according to the real-time data input.

Global SEP atmospheric radiation exposure is obtained from a pre-computed database. The ambient dose equivalent rates are calculated on a fixed 2-D grid in atmospheric depth and cutoff rigidity. The atmospheric depth grid extends from zero to $1300 \mathrm{~g} / \mathrm{cm}^{2}$, and the cutoff rigidity grid extends from zero to $19 \mathrm{GV}$. Both grids have non-uniform spacing with the highest number of grid points weighted toward low cutoff rigidities and tropospheric atmospheric depths. The real-time cutoff rigidities are computed on a $2.5 \mathrm{x}$ 2.5 horizontal grid. The pre-computed ambient dose equivalent rates are interpolated to the real-time cutoff rigidity and atmospheric depth specified at each horizontal grid point. 
Figure 10 shows global snapshots of atmospheric ambient dose equivalent rates over the northern hemisphere polar region for the Halloween 2003 SEP event 3. The ambient dose equivalent rates are shown at three altitudes and for three different magnetic field models used in the cutoff rigidity simulations. The left column shows exposure rates using the IGRF field. The middle column shows exposure rates computed for a geomagnetically quiet time prior to the onset of SEP event 3 using the T05 field (October 28, 2003, 0002 UT). The right column shows the exposure rates using the T05 field at the peak of the geomagnetic storm (October 29, 2003, 2100 UT) during SEP event 3. A typical cruising altitude for a commercial high-latitude flight is $11 \mathrm{~km}$. Overlaid on the $11 \mathrm{~km}$ ambient dose equivalent rate altitude surface are great circle routes for three representative highlatitude commercial flights: New York, New York (JFK) to London, England (LHR); Chicago, Illinois (ORD) to Stockholm, Sweden (ARN), and a combination of two great circle routes from Chicago, Illinois (ORD) to Beijing, China (PEK).

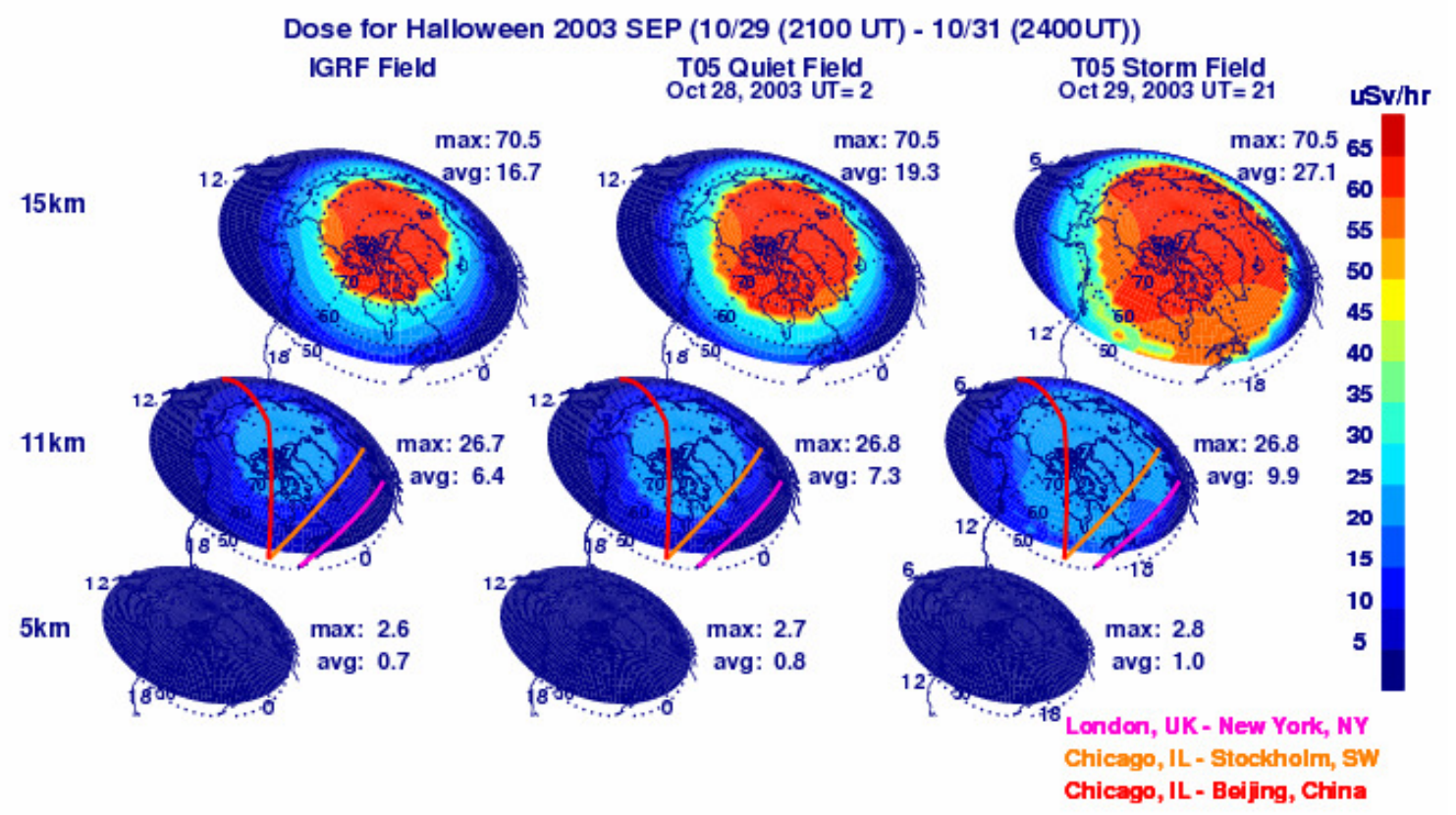

Figure 10: Ambient dose equivalent rates computed during the Halloween 2003 SEP event 3. The three columns correspond to exposure rates calculated using the geomagnetic cutoff rigidities and magnetic field models shown inError! Reference source not found.. The three rows are exposure rates calculated at different altitudes. In each graph, the hemispheric average ambient dose equivalent rate (uSv/hr) is indicated by the value next to "avg". The maximum exposure rate is indicated by the value next to "max". See text for definition of "avg" and "max". Also shown are the magnetic latitude circles and the meridians at $0,6,12$, and 18 magnetic local time.

There are a number of striking features to be noted from Figure 10. First, the representation of the geomagnetic field has a significant influence on SEP atmospheric radiation exposure. Comparing the left and middle columns of Figure 10 shows that even 
during geomagnetically quiet periods, the magnetospheric magnetic field weakens the overall geomagnetic field with a concomitant increase in radiation levels. This is seen as a broadening of the open-closed magnetospheric boundary in the T05 quiet field compared to the IGRF field. The cutoffs are zero in the region of open geomagnetic field lines. Thus, ambient dose equivalent rates based on the IGRF field are underestimated even for magnetically quiet times. During strong geomagnetic storms, as shown in the third column of Figure 7, the area of open field lines are broadened further, bringing large exposure rates to much lower latitudes. Ambient dose equivalent rates predicted using the IGRF model during a large geomagnetic storm can be significantly underestimated. The expansion of the polar region high exposure rates to lower latitudes, due to geomagnetic effects, is quantified by calculating hemispheric average ambient dose equivalent rates from $45 \mathrm{~N}$ to the pole. This is denoted by "avg" in Figure 10 . At $11 \mathrm{~km}$, there is roughly a $14 \%$ increase in the global-average ambient dose equivalent rate using T05 quiet-field compared to IGRF. During the geomagnetic storm, there is a 55\% increase in the global-average ambient dose equivalent rate using T05 storm-field compared to IGRF.

A second important feature to note in Figure 10 is the strong altitude dependence due to atmospheric shielding. The exposure rates are very low at $5 \mathrm{~km}$, independent of geomagnetic field model used. At $15 \mathrm{~km}$, the exposure rates are significantly higher than at $11 \mathrm{~km}$. Figure 10 shows that the SEP ambient dose equivalent rates increase (decrease) exponentially with increasing (decreasing) altitude. The SEP exposure rate altitude dependence is a fortunate feature for the aviation community, since radiation exposure can be significantly reduced by descending to lower altitudes. Private business jets will receive more radiation exposure than commercial aircraft if mitigation procedures are not taken, since business jet cruising altitudes are roughly $12-13 \mathrm{~km}$. The altitude dependence of the SEP exposure rates are quantified in Figure 10 by showing the maximum ambient dose equivalent rate at each altitude, which is the exposure rate at zero cutoff rigidity (i.e., in the polar region of open geomagnetic field lines). The maximum is denoted "max" in Figure 10. The exposure rate increases on average by $\sim 70 \%$ per km between 5 $\mathrm{km}$ and $11 \mathrm{~km}$. Between $11 \mathrm{~km}$ and $15 \mathrm{~km}$, the exposure rate increases on average by approximately $\sim 60 \%$ per $\mathrm{km}$.

Figure 11 shows the cutoff rigidities and ambient dose equivalent rates for the three representative high-latitude flights mentioned previously. The left column is the cutoff rigidities along the flight paths and the right column are the corresponding ambient dose equivalent rates along the flight paths. The cutoff rigidities include both latitude and time-dependent variations along the flight paths. The variations of the exposure rates along the flight paths include latitudinal variations in both atmospheric depth and cutoff rigidity. The temporal variations in cutoff rigidity also map into the variations of the exposure rates along the flight path. The top row shows results for the LHR-JFK flight, while the middle and bottom rows show results for the ORD-ARN and ORD-PEK flights, respectively. Each panel in Figure 11 shows cutoff rigidities and corresponding ambient dose equivalent rates using the IGRF field (green lines) and T05 storm-field (red lines) in the cutoff calculations. The largest differences in flight-path cutoff rigidities between IGRF and T05 storm-field models are for the LHR-JFK flight. The entire LHR-JFK flight path is near the magnetosphere open-closed boundary and is most sensitive to 
perturbations in cutoff rigidity due to geomagnetic effects. Consequently, the exposure rates along the LHR-JFK flight are most sensitive to geomagnetic effects. The ORD-PEK polar route is the least sensitive to geomagnetic suppression of the cutoff rigidity, since most of the flight path is across the polar cap region with open geomagnetic field lines. The influence of geomagnetic storm effects on the ORD-ARN flight is intermediate between a typical polar route and a flight along the North Atlantic corridor between the US and Europe.
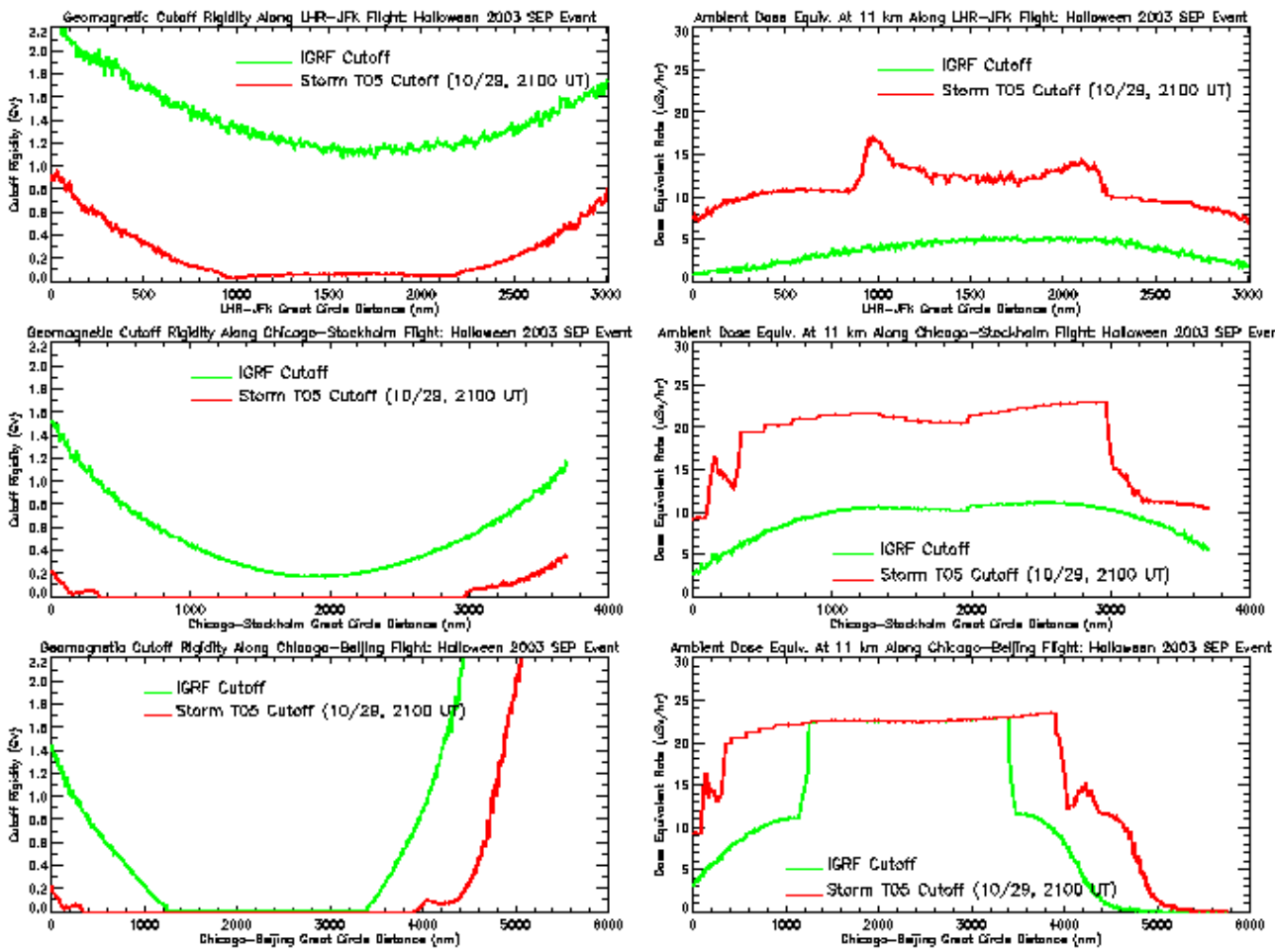

Figure 11: Geomagnetic cutoff rigidities (left column) and ambient dose equivalent rates (right column) calculated during Halloween 2003 SEP event 3 along three representative flight paths for a cruising altitude of $11 \mathrm{~km}$. The green line represents cutoff rigidities and exposure rates calculated using the IGRF model. The red lines represent cutoffs and exposure rates computed using the T05 model during the period of largest geomagnetic activity of event 3 . Note that the ambient dose equivalent rate calculated using the IGRF model for the LHR-JFK flight is scaled by a factor of two.

The total ambient dose equivalent along the three representative high-latitude flight paths is given in Table 3. The first three columns show total ambient dose equivalent computed from the three models of the geomagnetic field used in this study: IGRF, T05 quiet-field, and T05 storm-field. The last three columns show various ratios between the total ambient dose equivalent computed from the different geomagnetic field models. There are three major points to be noted from these results. One, the total ambient dose equivalent predicted for the ORD-PEK polar route for SEP event 3 during the Halloween 2003 storm reaches $22 \%$ of ICRP public/prenatal effective dose limit of $1 \mathrm{mSv}$. Two, 
using the IGRF field to compute the cutoff rigidity can underestimate the total ambient dose equivalent from $\sim 40 \%$ for polar routes to over a factor of three for flights along the North Atlantic corridor. Third, even for SEP events without an accompanying geomagnetic storm, using the IGRF field in cutoff rigidity simulations can underestimate the total ambient dose equivalent by roughly $30 \%$ for US flights into Europe.

In this initial study we have conducted an analysis of atmospheric ionizing radiation exposure associated with a high-energy SEP event during the Halloween 2003 storm period. The two main objectives of this analysis are the following: (1) provide an estimate of the exposure received on representative high-latitude commercial flights during the high-energy SEP event, and (2) to diagnose the influence of geomagnetic storm effects on SEP atmospheric radiation exposure. High-latitude flight paths are the routes most susceptible to significant SEP radiation exposure, since the cutoff rigidity rapidly approaches zero near the magnetosphere open-closed boundary.

The result from our first objective is that the radiation exposure during a representative polar flight was $22 \%$ of the ICRP public/prenatal limit. SEP exposure rates increase (decrease) exponentially with increasing (decreasing) altitude. Thus, SEP aircraft radiation exposure can be significantly reduced by descending to lower altitudes. Business jet cruising altitudes are higher than commercial aircraft. Consequently, private jets flying similar high-latitude routes as the commercial airlines will receive substantially more radiation if mitigation procedures are not enacted. NAIRAS real-time radiation exposure rate predictions during SEP events will enable the aviation community to make informed decisions concerning radiation risk evaluation and reduction.

To achieve our second objective of diagnosing the geomagnetic storm effects on SEP radiation exposure, we calculated the atmospheric ambient dose equivalent rates using event-averaged incident SEP proton and alpha fluence spectra and a static atmospheric depth-altitude relation, while the cutoff rigidity was calculated both statically and dynamically. The static cutoff rigidities were simulated using the IGRF field. The dynamic cutoff rigidities were simulated using the T05 field, which was allowed to respond to the real-time solar wind and IMF conditions. The dynamic cutoff rigidities were computed during a geomagnetically quiet period prior to the high-energy SEP event and during the peak of the geomagnetic storm associated with the high-energy SEP event. The key results of this study are as follows. One, ignoring solar wind-magnetosphere interactions during a strong geomagnetic storm in the calculation of cutoff rigidity can underestimate the total exposure by approximately $40 \%$ to over a factor of three. Two, even during geomagnetically quiet conditions, ignoring solar wind-magnetosphere interactions in the computed cutoff rigidities can underestimate the total exposure for flights along the North Atlantic corridor by roughly 30\%. To achieve more accurate assessments of aircraft radiation exposure, the magnetospheric influence on the cutoff rigidities must be included routinely in atmospheric radiation exposure predictions.

Our future efforts will build upon this work in four ways. One, we will study directional effects on the cutoff rigidities and subsequent radiation exposure rates. Two, we will model the aircraft fuselage and full body organ and tissue exposure. Third, we will allow 
the SEP fluence and atmospheric pressure to vary with time according to the real-time input data. Fourth, we will analyze additional storm periods to further quantify the relative contributions of SEP fluence, geomagnetic activity, and meteorological variability on atmospheric radiation exposure.

Table 1: data sources hosted on the SET operational server

\begin{tabular}{|c|c|c|}
\hline $\begin{array}{l}\text { 1-minute ACE/SWPAM } \\
\text { Data }\end{array}$ & Level 3 & $\begin{array}{l}\text { 1-minute ACE/SWPAM } \\
\text { Plot }\end{array}$ \\
\hline Hourly Kyoto Dst Data & Level 2 & Hourly Kyoto Dst Plot \\
\hline $\begin{array}{l}\text { 5-minute GOES-11 EPS } \\
\text { (P-channels) Data }\end{array}$ & Level 2 & $\begin{array}{l}\text { 5-minute GOES-11 EPS } \\
\text { (P-channels) Plot }\end{array}$ \\
\hline $\begin{array}{l}\text { 5-minute GOES-NN } \\
\text { Particle Data }\end{array}$ & Level 3 & $\begin{array}{l}\text { 5-minute GOES-NN } \\
\text { Particle Plot }\end{array}$ \\
\hline $\begin{array}{l}\text { Deprecated: 5-minute } \\
\text { GOES-11 Particle Data }\end{array}$ & Deprec. & $\begin{array}{l}\text { Deprecated: 5-minute } \\
\text { GOES-11 Particle Plot }\end{array}$ \\
\hline 3-Hour Ap Data & Level 2 & 3-Hour Ap Plot \\
\hline \multirow[t]{2}{*}{$\begin{array}{l}\text { Re-processed } \\
\text { NCAR/NCEP Air } \\
\text { Temperature Data }\end{array}$} & \multirow[t]{2}{*}{ Deprec. } & $\begin{array}{l}\text { Re-processed } \\
\text { NCAR/NCEP Air } \\
\text { Temperature Plot (contour, } \\
500 \mathrm{mb} \text { ) }\end{array}$ \\
\hline & & $\begin{array}{l}\text { Re-processed } \\
\text { NCAR/NCEP Air } \\
\text { Temperature Plot (color, } \\
500 \mathrm{mb} \text { ) }\end{array}$ \\
\hline \multirow[t]{2}{*}{$\begin{array}{l}\text { Current NCAR/NWS Air } \\
\text { Temperature Data (zip) }\end{array}$} & \multirow[t]{2}{*}{ Level 2} & $\begin{array}{l}\text { Current NCAR/NWS Air } \\
\text { Temperature Plot (contour, } \\
500 \mathrm{mb} \text { ) }\end{array}$ \\
\hline & & $\begin{array}{l}\text { Current NCAR/NWS Air } \\
\text { Temperature Plot (color, } \\
500 \mathrm{mb} \text { ) }\end{array}$ \\
\hline $\begin{array}{l}\text { Current NCAR/NWS } \\
\text { Geopotential Height Data } \\
\text { (zip) }\end{array}$ & Level 2 & None \\
\hline $\begin{array}{l}\text { 1-minute IEP SAS } \\
\text { Neutron Data }\end{array}$ & Level 2 & $\begin{array}{l}\text { 1-minute IEP SAS Neutron } \\
\text { Plot }\end{array}$ \\
\hline
\end{tabular}


Table 2: ACE/EPAM and GOES/SEM detectors used to derive SEP fluence spectra

\begin{tabular}{|c|c|c|c|}
\hline Particle & $\begin{array}{c}\text { Channel } \\
\text { Designation }\end{array}$ & $\begin{array}{c}\text { Energy Range } \\
(\mathrm{MeV})\end{array}$ & Instrument \\
\hline Proton & P3' & $0.114-0.190$ & LEMS120 (diff) \\
\hline Proton & P4' & $0.190-0.310$ & LEMS120 (diff) \\
\hline Proton & P5 & $0.310-0.580$ & LEMS120 (diff) \\
\hline Proton & P6' & $0.580-1.050$ & LEMS120 (diff) \\
\hline Proton & P7' & $1.050-1.890$ & LEMS120 (diff) \\
\hline Proton & P8' & $1.890-4.750$ & LEMS120 (diff) \\
\hline Proton & $\mathrm{P} 2$ & $4-9$ & EPS (diff) \\
\hline Proton & P3 & $9-15$ & EPS (diff) \\
\hline Proton & $\mathrm{P} 4$ & $15-40$ & EPS (diff) \\
\hline Proton & P5 & $40-80$ & EPS (diff) \\
\hline Proton & P6 & $80-165$ & EPS (diff) \\
\hline Proton & P7 & $165-500$ & EPS (diff) \\
\hline Proton & $\mathrm{P} 8$ & $350-420$ & HEPAD (diff) \\
\hline Proton & P9 & $420-510$ & HEPAD (diff) \\
\hline Proton & $\mathrm{P} 10$ & $510-700$ & HEPAD (diff) \\
\hline Proton & $\mathrm{I} 3-\mathrm{I} 2$ & $5-10$ & EPS $(>10->5)$ \\
\hline Proton & I4-I3 & $10-30$ & $\operatorname{EPS}(>30->10)$ \\
\hline Proton & I5-I4 & $30-50$ & EPS $(>50->30)$ \\
\hline Proton & I6-I5 & $50-60$ & EPS $(>60->50)$ \\
\hline Proton & I7-I6 & $60-100$ & $\operatorname{EPS}(>100->60)$ \\
\hline Alpha & A1 & $4-10$ & EPS (diff) \\
\hline Alpha & $\mathrm{A} 2$ & $10-21$ & EPS (diff) \\
\hline Alpha & A3 & $21-60$ & EPS (diff) \\
\hline Alpha & A4 & $60-150$ & EPS (diff) \\
\hline Alpha & A5 & $150-250$ & EPS (diff) \\
\hline Alpha & A6 & $300-500$ & EPS (diff) \\
\hline
\end{tabular}

Table 3: Total ambient dose equivalent using different geomagnetic cutoff models

\begin{tabular}{|l|c|c|c|c|c|c|}
\hline Flight Path & $\begin{array}{c}\text { Dose Eq. } \\
\text { T05S } \\
(\mathbf{m S v})\end{array}$ & $\begin{array}{c}\text { Dose Eq. } \\
\text { T05Q } \\
(\mathbf{m S v})\end{array}$ & $\begin{array}{c}\text { Dose Eq. } \\
\text { IGRF } \\
(\mathbf{m S v})\end{array}$ & $\begin{array}{c}\text { Dose Ratio } \\
\text { T05S/IGRF }\end{array}$ & $\begin{array}{c}\text { Dose Ratio } \\
\text { T05S/T05Q }\end{array}$ & $\begin{array}{c}\text { Dose Ratio } \\
\text { T05Q/IGRF }\end{array}$ \\
\hline JFK-LHR & 0.064 & 0.027 & 0.021 & 3.05 & 2.37 & 1.29 \\
\hline ORD-ARN & 0.158 & 0.105 & 0.078 & 2.03 & 1.50 & 1.35 \\
\hline ORD-PEK & 0.226 & 0.190 & 0.160 & 1.41 & 1.19 & 1.19 \\
\hline
\end{tabular}




\section{References}

Brandt, S. (1999), Data Analysis, Statistical and Computational Methods for Scientists and Engineers, Springer-Verlag, New York.

Ellison, D. C., and R. Ramaty, Shock acceleration ofelectrons and ions in solar flares (1985), Astrophys. J., 298, 400-408.

Gold, R. E., et al. (1998), Electron, Proton, and Alpha Monitor on the Advanced Composition and Explorer satellite, Space Sci. Rev., 86, 541.

Haggerty, D. K., E. C. Roelof, G. C. Ho, and R. E. Gold (2006), Quantitative comparison of ACE/EPAM data from different detector heads: Implications for NOAA RTSE users, Adv. Space Res., 38, 995-1000.

Kalnay, et al. (1996), The NCAR/NCEP 40-year reanalysis project, Bull. Amer. Meteor. Soc., 77, 437-470.

Kress, B. T., M. K. Hudson, K. L. Perry, and P. L. Slocum (2004), Dynamic modeling of geomagnetic cutoff for the 23-24 November 2001 solar energetic particle event, Geophy. Res. Lett., 31, L04808, doi:10.1029/2003GL018599.

Langlais, B., and M. Mandea (2000), An IGRF candidate geomagnetic field model for epoch 2000 and a secular variation model for 2000-2005, Earth Planets Space, 57, 1137-1148.

Lyon, J. G., J. A. Fedder and C. M. Mobarry (2004), The Lyon-Fedder-Mobarry (LFM) global MHD magnetospheric simulation code, J. Atmos. Solar-Terr. Phys., 66, 13331350.

Mewaldt, R. A., C. M. S. Cohen, A. W. Labrador, R. A. Leske, G. M. Mason, M. I. Desai, M. D. Looper, J. E. Mazur, R. S. Selesnick, and D. K. Haggerty (2005), Proton, helium, and electron spectra during the large solar particle events of OctoberNovember 2003, J. Geophys. Res., 110, A09S10, doi:10.1029/2005JA011038.

Onsager, T. G., et al. (1996), Operational uses of the GOES energetic particle detectors, in GOES-8 and Beyond, edited by E. R. Washwell, SPIE Conf. Proc., 2812, 281-290.

Picone, J. M., A. E. Hedin, D. P. Drob, and A. C. Aikin (2002), NRLMSIS-00 empirical model of the atmosphere: Statistical comparisons and scientific issues, J. Geophys. Res., 107(A12), 1468, doi:10/1029/2002JA009430.

Slaba, T. C., S. R. Blattnig, and S. K. Aghara (2008), Coupled neutron transport for HZETRN, submitted to Adv. Space Res.

Toffoletto, F. R., S. Sazykin, R. W. Spiro, R. A. Wolfand J. G. Lyon (2004), RCM meets LFM: initial results of one-way coupling, J. Atmos. Solar-Terr. Phys., 66, 1361-1370.

Tsyganenko, N. A., and N. I. Sitnov (2005), Modeling the dynamics of the inner magnetosphere during strong geomagnetic storms, J. Geophy. Res., 110, A03208, doi:10.1029/2004JA010798.

Wang, W., T. L. Kileen, A. G. Burns, and R. G. Roble (1999), A high resolution, three dimensional, time dependent nested grid model of the coupled thermosphereionosphere, J. Atmos. Solar-Terr. Phys., 61, 385-397. 